Noname manuscript No.

(will be inserted by the editor)

\title{
Combining linear and fast diffusion in a nonlinear elliptic equation
}

\author{
Willian Cintra • Cristian Morales \\ Rodrigo • Antonio Suárez
}

Received: date / Accepted: date

\begin{abstract}
In this paper we analyse an elliptic equation that combines linear and nonlinear fast diffusion with a logistic type reaction function. We prove existence and non-existence results of positive solutions using bifurcation theory and sub-supersolution method. Moreover, we apply variational methods to obtain a pair of ordered positive solutions.
\end{abstract}

Keywords Non-linear diffusion · Bifurcation · Sub-supersolution method . Variational Methods

Mathematics Subject Classification (2000) MSC 35B32 · 35J20 • $35 \mathrm{~J} 25 \cdot 35 \mathrm{~J} 60$

\section{Introduction}

In this paper we study the set of positive solutions of the following elliptic problem with nonlinear diffusion

$$
\begin{cases}-\Delta\left(u+a(x) u^{r}\right)=\lambda u-b u^{p} & \text { in } \Omega, \\ u=0 & \text { on } \partial \Omega,\end{cases}
$$

W. Cintra

Universidade Federal do Pará, Faculdade de Matemática

CEP: 66705-110 Belm - Pa, Brazil

E-mail: willian_matematica@hotmail.com

C. Morales-Rodrigo

Dpto. de Ecuaciones Diferenciales y Análisis Numérico

Fac. de Matemáticas, Univ. de Sevilla

Calle Tarfia s/n - Sevilla Spain

E-mail: cristianm@us.es

\section{A. Suárez}

Dpto. de Ecuaciones Diferenciales y Análisis Numérico

Fac. de Matemáticas, Univ. de Sevilla

Calle Tarfia s/n - Sevilla Spain

E-mail: suarez@us.es 
where $\Omega$ is a bounded and smooth domain of $\mathbb{R}^{N}, N \geq 1, \lambda \in \mathbb{R}, b \geq 0,0<$ $r<1<p$ and $a: \Omega \rightarrow[0, \infty)$ is a non-trivial regular function that can vanish on regions of $\Omega$. Thus, we will denote by

$$
\Omega_{a+}:=\{x \in \Omega ; a(x)>0\}
$$

and

$$
\Omega_{a 0}:=\Omega \backslash \bar{\Omega}_{a+} .
$$

Once that $r<1$, equation (1) provides us with the steady states of a porous medium equation where diffusion is linear in $\Omega_{a 0}$ and fast in $\Omega_{a+}$. Thus, in the context of population dynamics, $\Omega$ represents an habitat, $u(x)$ the density of the population of a species at $x \in \Omega$ and $-\Delta\left(u+a(x) u^{r}\right)$ describes the diffusion of the species, that is, the spacial movement, which is fast in some region of $\Omega$ $\left(\Omega_{a+}\right)$ and linear (or simple) in other $\left(\Omega_{a 0}\right)$. The function $\lambda u-b u^{p}$ is called logistic reaction term and, from biological point of view, $\lambda$ the intrinsic rate of natural increase of the species and $b$ denotes the maximum density supported locally by resources available, that is, the carrying capacity.

In particular, when $a \equiv 0$ in $\Omega$ (i.e., $\Omega_{a 0}=\Omega$ ), (1) reduces to the classical linear eigenvalue problem for the Laplacian operator under Dirichlet boundary conditions in $\Omega$ if $b=0$ and the classical logistic equation with linear diffusion if $b>0$. Subsequently, for any potential $V \in L^{\infty}(\Omega)$, we shall denote by $\lambda_{1}[-\Delta+V ; \Omega]$ the principal eigenvalue of $-\Delta+V$ in $\Omega$ under homogeneous Dirichlet boundary conditions. By simplicity, when $V \equiv 0$, we will denote

$$
\lambda_{1}=\lambda_{1}[-\Delta ; \Omega] \text {. }
$$

Thus, in the case $a=b=0$, according to the classical eigenvalue theory, (1) possesses a positive solution if, and only, if $\lambda=\lambda_{1}$. Actually, in such case, all positive solutions are the vector space generated by the principal eigenfunction. The study of case $b>0$ began with works of [6]. In this paper, the authors proved that there exists a unique positive solution if, and only if, $\lambda>\lambda_{1}$ and this positive solution attracts all the positive solution of the associated parabolic problem (see also [5], [11]). Hence, since the case $a \equiv 0$ is well-know, in this paper we consider only the $\Omega_{a 0} \neq \Omega$.

When $\Omega_{a 0} \neq \emptyset$, another eigenvalue problem plays an important role on the existence of positive solutions of (1). Specifically, the problem

$$
\begin{cases}-\Delta u=\lambda \mathcal{X}_{\Omega_{a 0}} u & \text { in } \Omega, \\ u=0 & \text { on } \partial \Omega .\end{cases}
$$

The existence of the principal eigenvalue of this problem is guaranteed by, for instance, [7] and [10]. Actually, denoting by $\lambda_{a 0}$ the principal eigenvalue of (2), it is given by the following variational characterization

$$
\lambda_{a 0}=\min _{\varphi \in H_{0}^{1}(\Omega) \backslash\{0\}} \frac{\|\varphi\|_{H_{0}^{1}}^{2}}{|\varphi|_{L^{2}\left(\Omega_{a 0}\right)}^{2}} .
$$



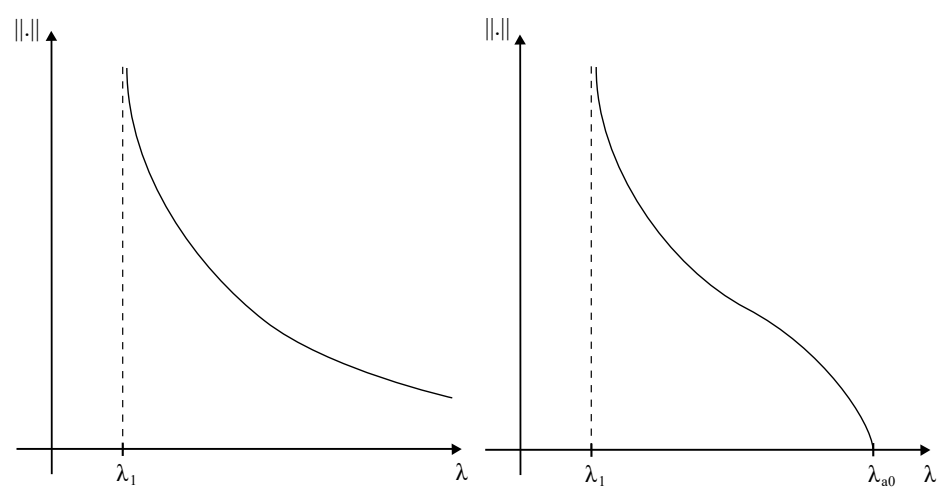

Fig. 1 Bifurcation diagrams in the case $b=0$ for $\Omega_{a 0}=\emptyset$ and $\Omega_{a 0} \neq \emptyset$, respectively.

This eigenvalue appears in problems that combine other types of nonlinear diffusion. For instance, [8] the authors analyzed the following problem

$$
\begin{cases}-\Delta\left(u^{m(x)}\right)=\lambda u & \text { in } \Omega, \\ u=0 & \text { on } \partial \Omega,\end{cases}
$$

where $m$ is a regular function with $m>1$ in a smooth subdomain $\Omega_{m}$ of $\Omega$ with $\bar{\Omega}_{m} \subset \Omega$ and $m \equiv 1$ in $\Omega \backslash \Omega_{m}$, that is, there exists a zone of linear diffusion, $\Omega \backslash \bar{\Omega}_{m}$, and a zone of nonlinear diffusion, $\Omega_{m}$. The authors show that (4) possesses a positive solutions if, and only if, $\lambda \in\left(0, \lambda_{m}\right)$, where $\lambda_{m}$ is the principal eigenvalue of (2) with $\Omega \backslash \Omega_{m}$ instead of $\Omega_{a 0}$. In fact, $\lambda=0$ is a bifurcation point from the trivial solution and $\lambda_{m}$ is a bifurcation point from infinity.

To emphasize the dependence of the parameter $\lambda$, we will refer to (1) as (1) $\lambda$. Thus, defining $\lambda_{a 0}=\infty$ if $\Omega_{a 0}=\emptyset$, our first main result is the following:

Theorem 1 If $b=0$ in $\Omega$, then $(1)_{\lambda}$ possesses a positive solution if, and only if, $\lambda \in\left(\lambda_{1}, \lambda_{a 0}\right)$. Moreover, any family of positive solutions $u_{\lambda}$ of $(1)_{\lambda}$ satisfies

$$
\lim _{\lambda \rightarrow \lambda_{1}}\left\|u_{\lambda}\right\|_{0}=\infty
$$

and

$$
\lim _{\lambda \rightarrow \lambda_{a 0}}\left\|u_{\lambda}\right\|_{0}=0 \quad \text { if } \lambda_{a 0}<\infty .
$$

In Figure 1 we have represented the corresponding bifurcation diagram of positive solutions of $(1)_{\lambda}$ with $b=0$. For the case $b>0$ the bifurcation from infinity disappears, in fact, we have

Theorem 2 If $b>0$, consider

$$
\Lambda_{b}=\left\{\lambda \in \mathbb{R} ;(1)_{\lambda} \text { has a positive solution }\right\} .
$$

Then $\Lambda_{b} \neq \emptyset$ and denoting by $\lambda^{*}(b)=\inf \Lambda_{b}$, we have $\lambda_{1}<\lambda^{*}(b) \leq \lambda_{a 0}$. Moreover, 

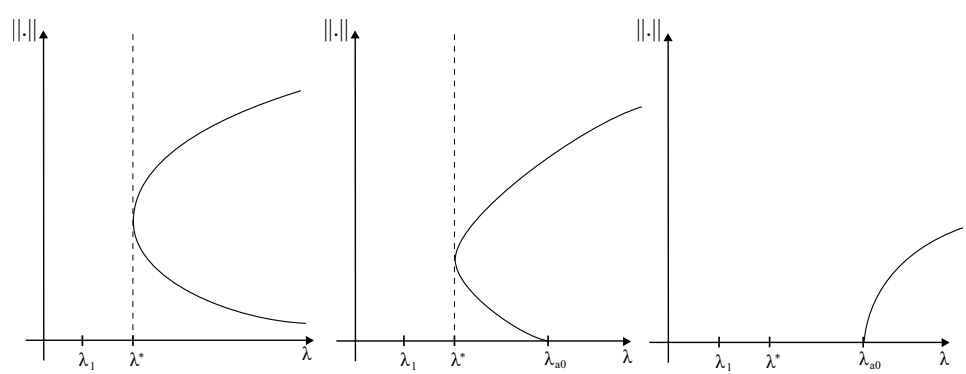

Fig. 2 Possible bifurcation diagrams. From the left to the right, the case $\Omega_{a 0}=\emptyset$. the case $\Omega_{a 0} \neq \emptyset$ with subcritical bifurcation and the case $\Omega_{a 0} \neq \emptyset$ with supercritical bifurcation.

(a) If $\Omega_{a 0}=\emptyset$, then $(1)_{\lambda}$ possesses a positive solution for all $\lambda \geq \lambda^{*}$.

(b) If $\Omega_{a 0} \neq \emptyset$, then $\lambda_{a 0}$ is a bifurcation point of (1) from the trivial solution and it is the only one for positive solutions. Furthermore, if the direction of the bifurcation is subcritical (resp. supercritical), then $(1)_{\lambda}$ possesses a positive solution for all $\lambda \geq \lambda^{*}$ (resp. $\left.\lambda>\lambda^{*}\right)$.

(c) In the case that $\lambda^{*}<\lambda_{a 0}$, then for each $\lambda \in\left(\lambda^{*}, \lambda_{a 0}\right),(1)_{\lambda}$ possesses two ordered positive solutions, that is, $w_{\lambda}$ and $v_{\lambda}$ positive solutions of $(1)_{\lambda}$ satisfying

$$
w_{\lambda}<v_{\lambda}
$$

Figure 2 shows some admissible situations within the setting of Theorem 2. We point out that in the case $b>0$ we do not have bifurcation from infinity and if $\Omega_{a 0}=\emptyset$ we also have not bifurcation from trivial solutions, and to conclude existence of positive solution we use the sub-supersolution method. For the case $\Omega_{a 0} \neq \emptyset$, in Proposition 4 we give conditions on $p, r, a$ and $b$ that provide us the direction of the bifurcation. This result show us an effect of the interaction between the fast diffusion $u+a(x) u^{r}$ and the logistic non-linearity $\lambda u-b u^{p}$. Specifically, if $1 / r<p$, then bifurcation from trivial solution is subcritical, while if $1 / r>p$ it is supercritical. In the case $1 / r=p, a$ and $b$ affect the direction of the bifurcation according to (20) and (21).

The next result gives us more information about the positive solutions with respect to the parameter $b$ :

Theorem 3 Assume $b>0$.

(a) For each $\lambda \geq \lambda^{*}(b)$, (1) possesses a maximal solution. That is, denoting it by $W_{\lambda(b)}$, then any positive solution, $w$, of (1) satisfies

$$
w \leq W_{\lambda(b)} .
$$

Moreover, if $\lambda^{*} \leq \mu<\lambda$, then $W_{\mu(b)}<W_{\lambda(b)}$.

(b) It holds

$$
\lambda^{*}(b) \rightarrow \lambda_{1} \quad \text { as } b \rightarrow 0 .
$$

(c) We have

$$
\lim _{b \rightarrow 0}\left\|W_{\lambda(b)}\right\|_{0}=\infty \quad \forall \lambda(b)>\lambda^{*}(b) .
$$




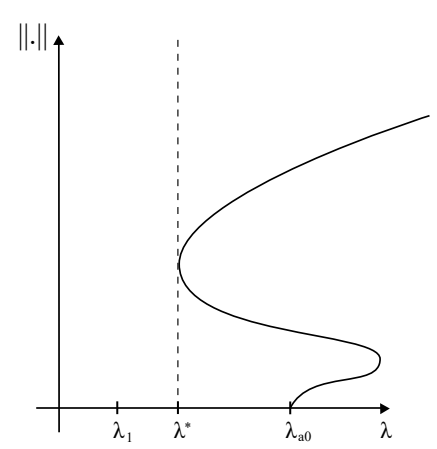

Fig. 3 An admissible bifurcation diagram when $b>0$ is small, $\Omega_{a 0} \neq \emptyset$ and the bifurcation is supercritical.

As a consequence, an interesting bifurcation diagram is admissible in case that $b$ is small and the bifurcation is supercritical. The paragraph (b) of Theorem 3 gives us that, for $b>0$ sufficiently small, $\lambda^{*}(b)<\lambda_{a 0}$. Then, if the bifurcation from the trivial solution is supercritical, the continuum of positive solutions which emanates from $\lambda_{a 0}$ goes to the right and, on the other hand, there exists positive solutions for $\lambda \in\left(\lambda^{*}(b), \lambda_{a 0}\right)$. Then, this leads us to a bifurcation diagram as in Figure 3.

The distribution of this paper is the following: in Section 2 we collect some useful previous results. Section 3 is dedicated to proof of Theorem 1 . Theorems 2 and 3 are proved in Section 4, with the exception of the existence of a second positive solution, which will be considered in Section 5 .

\section{Previous results}

We will present some basic results that will be used throughout this work. First, to deal with (1), we introduce the following change of variable

$$
I(x, u)=w=u+a(x) u^{r} \Leftrightarrow u=q(x, w)
$$

getting the following equivalent problem

$$
\begin{cases}-\Delta w=\lambda q(x, w)-b q(x, w)^{p} & \text { in } \Omega, \\ w=0 & \text { on } \partial \Omega .\end{cases}
$$

Since we are interested in positive solutions of $(1)_{\lambda}$, we can define

$$
q(x, s)=0, \quad \forall x \in \Omega, s \leq 0 .
$$

Thus, by the Strong Maximal Principle, any non-trivial solution of $(1)_{\lambda}$ is in fact strictly positive. Hence $u>0$ is a positive solution of $(1)_{\lambda}$ if, and only if, $w=u+a(x) u^{r}$ is a positive solution of (9). Therefore, we analyze the equivalent problem (9). Again, we will refer to (9) as $(9)_{\lambda}$.

Let us prove some useful properties of the function $q(x, s)$ 
Lemma 1 1. For each $x \in \Omega$, the map $s \mapsto q(x, s), s \geq 0$ is of class $\mathcal{C}^{1}$. 2. For all $x \in \Omega$, the map

$$
s \mapsto \frac{q(x, s)}{s} \quad s \geq 0,
$$

is non-decreasing and satisfies

$$
\begin{gathered}
\mathcal{X}_{\Omega_{a 0}}(x) s \leq q(x, s) \leq s \quad \forall x \in \Omega, \\
\lim _{s \rightarrow 0} \frac{q(x, s)}{s}=\mathcal{X}_{\Omega_{a 0}}(x)= \begin{cases}0 & \text { if } a(x)>0, \\
1 & \text { if } a(x)=0 .\end{cases}
\end{gathered}
$$

and

$$
\lim _{s \rightarrow \infty} \frac{q(x, s)}{s}=1
$$

3. For all $x \in \Omega$, the map

$$
s \mapsto \frac{q(x, s)^{p}}{s}
$$

is increasing and satisfies

$$
\lim _{s \rightarrow 0} \frac{q(x, s)^{p}}{s}=0
$$

and

$$
\lim _{s \rightarrow \infty} \frac{q(x, s)^{p}}{s}=+\infty
$$

Proof 1. Since $q(x, \cdot)$ is the inverse function of $I(x, s)=s+a(x) s^{r}$, we get

$$
q^{\prime}(x, s)=\frac{1}{1+r a(x) q(x, s)^{r-1}} .
$$

Therefore $q^{\prime}(x, s)$ is continuous in $(0, \infty)$. On the other hand,

$$
\lim _{s \rightarrow 0^{+}} q^{\prime}(x, s)=\lim _{s \rightarrow 0^{+}} \frac{1}{1+a(x) r q(x, s)^{r-1}}=\mathcal{X}_{\Omega_{a 0}}(x)=q^{\prime}(x, 0),
$$

showing the continuity at 0 .

2. Observe that

$$
I(x, q(x, s))=s=q(x, s)+a(x) q(x, s)^{r},
$$

and therefore

$$
\frac{q(x, s)}{s}=\frac{1}{1+a(x) q(x, s)^{r-1}},
$$

where we deduce (10). Moreover, since $s \mapsto q(x, s)$ is increasing and $r<1$, (15) provides that $q(x, s) / s$ is non-decreasing. 
To calculate the limits (11)-(12), observe that if $a(x)=0$ we have $q(x, s) / s$

$=1$ and it is immediate. If $a(x)>0$, using

$$
\lim _{s \rightarrow 0} q(x, s)=0 \quad \text { and } \quad \lim _{s \rightarrow \infty} q(x, s)=\infty,
$$

(15) gives

$$
\lim _{s \rightarrow 0} \frac{q(x, s)}{s}=0 \quad \text { and } \quad \lim _{s \rightarrow \infty} \frac{q(x, s)}{s}=1 .
$$

3. Analogously, observe that

$$
\frac{q(x, s)^{p}}{s}=\frac{1}{q(x, s)^{1-p}+a(x) q(x, s)^{r-p}} .
$$

By the monotonicity of $s \mapsto q(x, s)$ and since $r<1<p$, it follows that $q(x, s) / s$ is increasing in $s$, for all $x \in \Omega$. Moreover, letting $s \rightarrow 0$ and $s \rightarrow \infty$ in (16), yields to (13)-(14).

The following function will play a crucial role in our exposition

$$
\mu(\lambda):=\lambda_{1}\left[-\Delta-\lambda \mathcal{X}_{\Omega_{a 0}} ; \Omega\right], \quad \lambda \in \mathbb{R} .
$$

It is well defined because $-\lambda \mathcal{X}_{\Omega_{a 0}} \in L^{\infty}(\Omega)$ for all $\lambda \in \mathbb{R}$ and the next result provides some properties of this function and that will be useful throughout the work.

Proposition 1 The function $\mu$ defined in (17) is decreasing and possesses a unique zero, say $\lambda_{a 0}$. Moreover, $\mu(\lambda)>0$ if, and only if, $\lambda<\lambda_{a 0}$. Furthermore, it satisfies

$$
\lambda_{1}<\lambda_{a 0},
$$

and $\lambda_{a 0}$ is the principal eigenvalue of (2).

Proof Observe that, by the monotonicity of $\lambda_{1}\left[-\Delta-\lambda \mathcal{X}_{\Omega_{a 0}} ; \Omega\right]$ with respect of the potential, we get

$$
\lambda_{1}-\lambda<\mu(\lambda)<\lambda_{1}\left[-\Delta ; \Omega_{a 0}\right]-\lambda,
$$

consequently, $\mu(\lambda) \rightarrow-\infty$ as $\lambda \rightarrow+\infty$ and

$$
\lambda_{1}-\lambda_{a 0}<\mu\left(\lambda_{a 0}\right)=0 .
$$

Moreover, by $\underline{[9]}, \mu^{\prime}(\lambda)<0$ (see $\underline{10]}$ for further details). Therefore, since $\mu$ is a continuous function and $\mu(0)=\lambda_{1}[-\Delta ; \Omega]>0$, there exists a unique $\lambda_{a 0} \in \mathbb{R}$, such that $\mu\left(\lambda_{a 0}\right)=0$. Furthermore, since $\mu$ is decreasing, it follows that $\mu(\lambda)>0$ if, and only if, $\lambda<\lambda_{a 0}$.

Finally, note that

$$
\mu\left(\lambda_{a 0}\right)=\lambda_{1}\left[-\Delta-\lambda_{a 0} \mathcal{X}_{\Omega_{a 0}} ; \Omega\right]=0
$$

is equivalent to say that $\lambda_{a 0}$ is the principal eigenvalue of (2). 
Proof Observe that, by the monotonicity of $\lambda_{1}\left[-\Delta-\lambda \mathcal{X}_{\Omega_{a 0}} ; \Omega\right]$ with respect of the potential, we get

$$
\lambda_{1}-\lambda<\mu(\lambda)<\lambda_{1}\left[-\Delta ; \Omega_{a 0}\right]-\lambda,
$$

consequently, $\mu(\lambda) \rightarrow-\infty$ as $\lambda \rightarrow+\infty$ and

$$
\lambda_{1}<\mu(0) .
$$

Moreover, by [9], $\mu^{\prime}(\lambda)<0$ (see [10] for further details). Therefore, since $\mu$ is a continuous function and $\mu(0)=\lambda_{1}>0$, there exists a unique $\lambda_{a 0} \in \mathbb{R}$, such that $\mu\left(\lambda_{a 0}\right)=0$. Furthermore,

$$
\lambda_{1}-\lambda_{a 0}<\mu\left(\lambda_{a 0}\right)=0
$$

and, since $\mu$ is decreasing, it follows that $\mu(\lambda)>0$ if, and only if, $\lambda<\lambda_{a 0}$ and

Finally, note that

$$
\mu\left(\lambda_{a 0}\right)=\lambda_{1}\left[-\Delta-\lambda_{a 0} \mathcal{X}_{\Omega_{a 0}} ; \Omega\right]=0
$$

is equivalent to say that $\lambda_{a 0}$ is the principal eigenvalue of (2).

To end this section, we will study an auxiliary problem that will provide us the existence of a maximal solution to $(9)_{\lambda}$ and a priori bound for positive solutions of $(9)_{\lambda}$. Specifically, consider the problem

$$
\begin{cases}-\Delta w=\lambda w-b q(x, w)^{p} & \text { in } \Omega, \\ w=0 & \text { on } \partial \Omega .\end{cases}
$$

Proposition 2 (19) possesses a positive solution if, and only if $\lambda>\lambda_{1}$. Moreover, it is unique if it exists and we will denote it by $\theta_{\lambda}$ and

$$
\theta_{\mu} \leq \theta_{\lambda} \quad \text { if } \lambda_{1}<\mu \leq \lambda .
$$

Proof If $w>0$ is a solution of (19), then

$$
\lambda=\lambda_{1}\left[-\Delta+b q(x, w)^{p} / w ; \Omega\right]>\lambda_{1}[-\Delta ; \Omega]=\lambda_{1} .
$$

Consequently, $\lambda>\lambda_{1}$ is a necessary condition for the existence of positive solutions. Now, suppose $\lambda>\lambda_{1}$. To prove the existence of positive solution, observe that $\left(\varepsilon \varphi_{1}, K\right)$ is a pair of sub-supersolution of (19) for constants $\varepsilon>0$ small and $K>0$ large.

The uniqueness follows by Theorem 1 of [5], once that

$$
s \mapsto \lambda-b \frac{q(x, s)^{p}}{s}
$$

is decreasing for all $x \in \Omega$. Finally, the monotonicity with respect to $\lambda$ follows from the comparison principle.

Corollary 1 For any $\lambda \geq \mu>\lambda_{1}$, any positive solution $w_{\mu}$ of $(9)_{\mu}$ satisfies

$$
w_{\mu} \leq \theta_{\mu} \leq \theta_{\lambda} .
$$

Proof Just observe that $w_{\mu}$ is a subsolution of (19) and $K$ sufficiently large is a supersolution. Hence, by the uniqueness of solution of (19), necessarily

$$
w_{\mu} \leq \theta_{\mu} \leq \theta_{\lambda} .
$$


3 Case $b=0$.

This section is dedicated to study the case $b=0$. To this, we use bifurcation techniques. Thus, we consider the map $\Phi_{\lambda}: \mathcal{C}_{0}(\bar{\Omega}) \longrightarrow \mathcal{C}_{0}(\bar{\Omega})$ defined by

$$
\Phi_{\lambda}(w)=I-(-\Delta)^{-1}(\lambda q(x, w)),
$$

here $(-\Delta)^{-1}$ is the inverse of Laplace operator under homogeneous Dirichlet boundary condition. Observe that $w \in \mathcal{C}_{0}(\bar{\Omega})$ is a positive solution of (9) if, and only if, $\Phi_{\lambda}(w)=0$. Denoting by $\Sigma$ the closure of the set

$$
\left\{(\lambda, w) \in \mathbb{R} \times \mathcal{C}_{0}(\bar{\Omega}) \text { such that } \Phi_{\lambda}(w)=0, w \neq 0\right\},
$$

we get

Proposition 3 Suppose $b=0$ in $\Omega$,

1. If there exists a positive solution of $(9)_{\lambda}$, then $\lambda \in\left(\lambda_{1}, \lambda_{a 0}\right)$.

2. $\lambda_{1}$ is the unique bifurcation point from the infinity of positive solutions of

$(9)_{\lambda}$. Moreover, there exists a unbounded component $\Sigma_{\infty} \subset \Sigma$ such that

$$
\bar{\Sigma}_{\infty}=\left\{(\lambda, w) \text { with } w \neq 0 ; \quad\left(\lambda, \frac{w}{\|w\|_{0}^{2}}\right) \in \Sigma_{\infty}\right\} \cup\left\{\left(\lambda_{1}, 0\right)\right\}
$$

is connected and unbounded.

Proof 1. If $w>0$ is a solution of $(9)_{\lambda}$, we have

$$
\left\{\begin{array}{lr}
{\left[-\Delta-\lambda \frac{q(x, w)}{w}\right] w=0,} & \text { in } \Omega \\
w=0, & \text { on } \partial \Omega .
\end{array}\right.
$$

Using (10), we obtain

$$
0=\lambda_{1}\left[-\Delta-\lambda \frac{q(x, w)}{w} ; \Omega\right]>\lambda_{1}[-\Delta-\lambda ; \Omega]=\lambda_{1}-\lambda .
$$

In the case $\Omega_{a 0} \neq \emptyset$, using again (10), we derive that

$$
0=\lambda_{1}\left[-\Delta-\lambda \frac{q(x, w)}{w} ; \Omega\right]<\lambda_{1}\left[-\Delta-\lambda \mathcal{X}_{\Omega_{a 0}} ; \Omega\right]=\mu(\lambda) .
$$

By the properties of function $\mu$, it follows that $\lambda<\lambda_{a 0}$.

2. In view of (12) and since $f(\lambda, x, s):=\lambda q(x, s)$ satisfies $f(0, x, s) \equiv 0$ for all $x \in \Omega$ and $s \geq 0$, we can apply the Theorem 3.4 of $[3]$ and get the results.

Proof of Theorem 1:

By Proposition $32 ., \lambda_{1}$ is a bifurcation point of $(9)_{\lambda}$ from infinity and it is the only one for positive solutions. In order to prove the existence of solution for $\lambda \in\left(\lambda_{1}, \lambda_{a 0}\right)$, we will consider two cases: $\Omega_{a 0}=\emptyset$ and $\Omega_{a 0} \neq \emptyset$.

Case $\Omega_{a 0}=\emptyset$ : To conclude the results, it is sufficient to check the following: 
Claim: for all compact set $\Lambda \subset\left[\lambda_{1}, \infty\right)$ there exists $\varepsilon>0$ such that $(9)_{\lambda}$ has no positive solution with $(\lambda, w) \in \Lambda \times B_{\varepsilon}(0)$.

Indeed, because the global nature of $\Sigma_{\infty}$ implies that it is unbounded with respect to $\lambda$ and, since $(9)_{\lambda}$ has no positive solution for $\lambda<\lambda_{1}$ (Proposition $3)$, the result follows.

Let us prove the claim. Arguing by contradiction, there exists $\left(\lambda_{n}, w_{n}\right)$ a sequence of solutions of $(9)_{\lambda_{n}}$ such that $\lambda_{n} \in \Lambda$ for all $n \in \mathbb{N}$ and $\left\|w_{n}\right\|_{0} \rightarrow 0$. Since $\Lambda$ is compact, up to subsequence if necessary, we have

$$
\left(\lambda_{n}, w_{n}\right) \rightarrow\left(\lambda^{*}, 0\right) \quad \text { in } \mathbb{R} \times \mathcal{C}_{0}(\bar{\Omega})
$$

From (11) and previous limit we get that for all $\delta>0$, there exists $n_{\delta} \in \mathbb{N}$ such that

$$
\frac{q\left(x, w_{n}\right)}{w_{n}} \leq \delta \quad \forall n>n_{\delta} .
$$

Thus, since $\left(\lambda_{n}, w_{n}\right)$ is a solution of $(9)_{\lambda_{n}}$, we obtain

$$
0=\lambda_{1}\left[-\Delta-\lambda_{n} \frac{q\left(x, w_{n}\right)}{w_{n}} ; \Omega\right]>\lambda_{1}\left[-\Delta-\lambda_{n} \delta ; \Omega\right]=\lambda_{1}-\lambda_{n} \delta \quad \forall n>n_{\delta},
$$

that is,

$$
\lambda_{n} \delta>\lambda_{1} .
$$

Letting $n \rightarrow \infty$ and thanks to $\lambda_{n} \rightarrow \lambda^{*}<\infty$, the above inequality provides $\lambda_{1} \leq \lambda^{*} \delta$, for all $\delta>0$, which is a contradiction.

Case $\Omega_{a 0} \neq \emptyset$

In view of (11), we can apply Theorem 4.4 of [3] and obtain that $\lambda_{a 0}$ is a bifurcation point from the trivial solution of positive solutions, and it is the only one in $\mathbb{R}_{0}^{+}$. Furthermore, there exists an unbounded component $\Sigma_{0} \subset \Sigma$ meeting $\lambda_{a 0}$. Once that these bifurcation points are unique, we get

$$
\Sigma_{\infty}=\Sigma_{0} .
$$

As a consequence, by global nature of these continuum, we obtain that there exist positive solutions for all $\lambda \in\left(\lambda_{1}, \lambda_{a 0}\right)$.

\section{Case $b>0$}

In this section we will prove Theorems 2 and 3, except the existence of a second solution that will be treated in the next section.

First, denoting by $\varphi_{a 0}$ the principal positive eigenfunction associated to $\lambda_{a 0}$ with $\left\|\varphi_{a 0}\right\|_{0}=1$, we have the following result of existence and non-existence of positive solutions.

Proposition 4 1. If $(9)_{\lambda}$ possesses a positive solution, then $\lambda>\lambda_{1}$.

2. If $\Omega_{a 0} \neq \emptyset$, then $\lambda_{a 0}$ is a bifurcation point of (9) from the trivial solution and it is the only one for positive solutions. Furthermore, the bifurcation is 
(a) Subcritical if $1 / r<p$.

(b) Subcritical if $1 / r=p$ and

$$
\int_{\Omega_{a+}} \frac{\varphi_{a 0}^{p+1}}{a(x)^{p}}>b \int_{\Omega_{a 0}} \varphi_{a 0}^{p+1} .
$$

(c) Supercritical if $1 / r=p, a(x)^{-p} \in L^{1}\left(\Omega_{a+}\right)$ and

$$
\int_{\Omega_{a+}} \frac{\varphi_{a 0}^{p+1}}{a(x)^{p}}<b \int_{\Omega_{a 0}} \varphi_{a 0}^{p+1} .
$$

(d) Supercritical if $1 / r>p$.

3. There exists $\bar{\lambda}>\lambda_{1}$ such that $(9)_{\bar{\lambda}}$ has a positive solution

Proof The proof of first paragraph is similar to first one of Proposition 3. Thus, we will prove only 2 and 3 .

We prove first the second paragraph. If $\Omega_{a 0} \neq \emptyset$, by (11), we can apply the Theorem 4.4 of [3] to obtain that $\lambda_{a 0}$ is the only bifurcation point from the trivial solution. To conclude the direction of bifurcation we will apply the paragraphs (i) and (ii) of Theorem 4.4 of [3] and argue as follows. Denote

$$
g(\lambda, x, s):=\frac{\lambda q(x, s)-b q(x, s)^{p}-\lambda \mathcal{X}_{\Omega_{a 0}}(x) s}{s^{1-\sigma}},
$$

where $\sigma<0$ to be chosen later.

(a) If $1 / r<p$, we choose $\sigma=1-1 / r$. Thus, in $\Omega_{a+}$ we have

$$
\begin{aligned}
g(\lambda, x, s) & =\lambda \frac{\left(q(x, s)^{r}\right)^{1 / r}}{\left(q(x, s)+a(x) q(x, s)^{r}\right)^{1 / r}}-b \frac{\left(q(x, s)^{p r}\right)^{1 / r}}{\left(q(x, s)+a(x) q(x, s)^{r}\right)^{1 / r}} \\
& =\lambda \frac{1}{\left(q(x, s)^{1-r}+a(x)\right)^{1 / r}}-b \frac{1}{\left(q(x, s)^{1-p r}+a(x) q(x, s)^{(1-p) r}\right)^{1 / r}}
\end{aligned}
$$

and, therefore,

$$
\liminf _{(\lambda, s) \rightarrow\left(\lambda_{a 0}, 0^{+}\right)} g(\lambda, x, s)=\frac{\lambda_{a 0}}{a(x)^{1 / r}} \quad \text { in } \Omega_{a+} .
$$

On the other hand, in $\Omega_{a 0}$ we have

$$
g(\lambda, x, s)=\frac{\lambda s-b s^{p}-\lambda s}{s^{1 / r}}=-b s^{p-1 / r}
$$

and, since $1 / r<p$, we obtain that

$$
\liminf _{(\lambda, s) \rightarrow\left(\lambda_{a 0}, 0^{+}\right)} g(\lambda, x, s)=0 \quad \text { in } \Omega_{a 0} .
$$

Consequently,

$$
\underline{\mu}(x) \equiv \liminf _{(\lambda, s) \rightarrow\left(\lambda_{a 0}, 0^{+}\right)} g(\lambda, x, s) \geq 0
$$

and

$$
\int_{\Omega} \underline{\mu}(x) \varphi_{a 0}^{1 / r+1}>0 .
$$

Then, by Theorem 4.4 (i) of [3], the bifurcation of positive solutions at $\lambda=\lambda_{a 0}$ is subcritical. 
(b) If $1 / r=p$, we choose $\sigma=1-p$. Thus, in $\Omega_{a+}$, we have

$$
g(\lambda, x, s)=\lambda \frac{1}{\left(q(x, s)^{1-1 / p}+a(x)\right)^{p}}-b\left(\frac{q(x, s)}{s}\right)^{p} .
$$

Implying that

$$
\underline{\mu}(x) \equiv \liminf _{(\lambda, s) \rightarrow\left(\lambda_{a 0}, 0^{+}\right)} g(\lambda, x, s)=\frac{\lambda_{a 0}}{a(x)^{p}} \quad \text { in } \Omega_{a+} .
$$

On the other hand, in $\Omega_{a 0}$ we have

$$
g(\lambda, x, s)=\frac{\lambda s-b s^{p}-\lambda s}{s^{p}}=-b .
$$

Consequently,

$$
\underline{\mu}(x) \equiv \liminf _{(\lambda, s) \rightarrow\left(\lambda_{a 0}, 0^{+}\right)} g(\lambda, x, s)= \begin{cases}\frac{\lambda_{a 0}}{a(x)^{p}} & \text { if } x \in \Omega_{a+}, \\ -b & \text { if } x \in \Omega_{a 0} .\end{cases}
$$

Therefore, $\underline{\mu}(x) \geq-b$ and (20) is equivalent to

$$
\int_{\Omega} \underline{\mu}(x) \varphi_{a 0}^{p+1}>0 .
$$

Thus, by Theorem 4.4 (i) of [3], the bifurcation of positive solutions at $\lambda=\lambda_{a 0}$ is subcritical.

(c) Analogously to the previous case, for $\sigma=1-p$ we have

$$
\bar{\mu}(x) \equiv \limsup _{(\lambda, s) \rightarrow\left(\lambda_{a 0}, 0^{+}\right)} g(\lambda, x, s)= \begin{cases}\frac{\lambda_{a 0}}{a(x)^{p}} & \text { if } x \in \Omega_{a+}, \\ -b & \text { if } x \in \Omega_{a 0} .\end{cases}
$$

Once that $a(x)^{-p} \in L^{1}\left(\Omega_{a+}\right)$, we get $\bar{\mu} \in L^{1}(\Omega)$ and since (21) is equivalent to

$$
\int_{\Omega} \bar{\mu}(x) \varphi_{a 0}^{p+1}<0
$$

Theorem 4.4 (ii) of [3] implies that the bifurcation of positive solutions at $\lambda=\lambda_{a 0}$ is supercritical.

(d) If $1 / r>p$, we choose $\sigma=1-p$. Thus, in $\Omega_{a+}$, we have

$$
g(\lambda, x, s)=\lambda \frac{1}{\left(q(x, s)^{1-1 / p}+a(x) q(x, s)^{r-1 / p}\right)^{p}}-b\left(\frac{q(x, s)}{s}\right)^{p}
$$

and, since $1 / r>p$,

$$
\limsup _{(\lambda, s) \rightarrow\left(\lambda_{a 0}, 0^{+}\right)} g(\lambda, x, s)=0 \quad \text { in } \Omega_{a+} .
$$


On the other hand, in $\Omega_{a 0}$ we have

$$
g(\lambda, x, s)=\frac{\lambda s-b s^{p}-\lambda s}{s^{p}}=-b .
$$

Consequently,

$$
\bar{\mu}(x) \equiv \limsup _{(\lambda, s) \rightarrow\left(\lambda_{a 0}, 0^{+}\right)} g(\lambda, x, s)=-\mathcal{X}_{\Omega_{a 0}} b \in L^{1}(\Omega)
$$

and

$$
\int_{\Omega} \bar{\mu}(x) \varphi_{a 0}^{p+1}<0 .
$$

Then, by Theorem 4.4 (ii) of [3], the bifurcation of positive solutions at $\lambda=\lambda_{a 0}$ is supercritical.

To prove the third paragraph, note that the case $\Omega_{a 0} \neq \emptyset$ is a immediate consequence of the second paragraph.

If $\Omega_{a 0}=\emptyset$, then we can not apply the bifurcation theorem, thus we will use the method of sub-supersolution to prove the existence of positive solution for $\lambda>\lambda_{1}$ large.

To build the subsolution, denoting by $\varphi_{1}>0$, the eigenvalue associated to $\lambda_{1}$ with $\left\|\varphi_{1}\right\|_{0}=1$, it satisfies

$$
\begin{aligned}
\Delta\left(\varphi_{1}^{m}\right) & =m(m-1) \varphi_{1}^{m-2}\left|\nabla \varphi_{1}\right|^{2}+m \varphi_{1}^{m-1} \Delta \varphi_{1} . \\
& =m(m-1) \varphi_{1}^{m-2}\left|\nabla \varphi_{1}\right|^{2}-m \lambda_{1} \varphi_{1}^{m} .
\end{aligned}
$$

Therefore, $\underline{w}=\varphi_{1}^{m}$ is a subsolution of $(9)_{\lambda}$ provided that

$$
-\Delta\left(\varphi_{1}^{m}\right) \leq \lambda q\left(x, \varphi_{1}^{m}\right)-b q\left(x, \varphi_{1}^{m}\right)^{p} \quad \forall x \in \Omega,
$$

once that $q\left(x, \varphi_{1}^{m}\right)>0$ for all $x \in \Omega$, this inequality is equivalent to

$$
\frac{m \varphi_{1}^{m}}{q\left(x, \varphi_{1}^{m}\right)}\left((1-m) \frac{\left|\nabla \varphi_{1}\right|^{2}}{\varphi_{1}^{2}}+\lambda_{1}\right)+b q\left(x, \varphi_{1}^{m}\right)^{p-1} \leq \lambda \quad \forall x \in \Omega .
$$

Note that the term $b q\left(x, \varphi_{1}^{m}\right)$ is bounded. Let us show that the remaining terms are also bounded. Indeed, observe that

$$
(1-m) \frac{\left|\nabla \varphi_{1}\right|^{2}}{\varphi_{1}^{2}}+\lambda_{1} \leq 0
$$

provided that

$$
\left(\frac{\lambda_{1}}{m-1}\right)^{1 / 2} \leq \frac{\left|\nabla \varphi_{1}\right|}{\varphi_{1}}
$$

Since $\varphi_{1}=0$ and $\partial \varphi_{1} / \partial \eta<0$ in $\partial \Omega$, where $\eta=\eta(x)$ denote the outward normal derivative of $\varphi_{1}$ in the point $x \in \partial \Omega$, we can obtain $\delta>0$ such that

$$
\begin{aligned}
\Omega_{\delta}:= & \{x \in \Omega ; d(x, \partial \Omega) \leq \delta\} \subset \\
& \left\{x \in \Omega ;\left(\lambda_{1} /(m-1)\right)^{1 / 2} \leq\left|\nabla \varphi_{1}(x)\right| / \varphi_{1}(x)\right\} .
\end{aligned}
$$


As a consequence, (23) occurs for all $x \in \Omega_{\delta}$.

On the other hand, since

$$
M=\min _{x \in \Omega \backslash \Omega_{\delta}} \varphi_{1}^{m}(x)>0
$$

and the map $s \mapsto s / q(x, s)$ is non-increasing, it follows

$$
\frac{\varphi_{1}^{m}}{q\left(x, \varphi_{1}^{m}\right)} \leq \frac{M}{q(x, M)} \quad \forall x \in \Omega \backslash \Omega_{\delta} .
$$

Thus, thanks to (23) and (25), we get (22) for $\lambda$ large enough therefore $\underline{w}=\varphi_{1}^{m}$ is a subsolution of $(9)_{\lambda}$.

Now, let $K>0$ a positive constant. Then $\bar{w}=K$ is a supersolution of $(9)_{\lambda}$, provided that

$$
0=-\Delta K \geq \lambda q(x, K)-b q(x, K)^{p},
$$

which is equivalent to

$$
q(x, K)^{p-1} \geq \frac{\lambda}{b} .
$$

Hence, choosing $K$ satisfying (26) and $K>\varphi_{1}^{m}, \bar{w}=K$ is a supersolution of $(9)_{\lambda}$. Consequently, there exists a positive soution $w$ of $(9)_{\lambda}$ for $\lambda$ large, satisfying

$$
\varphi_{1}^{m} \leq w \leq K .
$$

Proof of Theorem 2 (b) and (c): Once that $b>0$ is fixed in this theorem, here we will denote $\lambda^{*}(b)$ simply by $\lambda^{*}$.

Thanks to Proposition 4 we already have that $\Lambda_{b} \neq \emptyset$ and $\lambda_{1} \leq \lambda^{*}<\infty$. With the notation $\lambda_{a 0}=\infty$ if $\Omega_{a 0}=\emptyset$, we can deal with paragraphs (b) and (c) simultaneously to show existence of positive solution for $\lambda>\lambda^{*}$.

Thus, if $\lambda>\lambda^{*}$, by definition of $\lambda^{*}$, we can get that there exists $\bar{\lambda}$ with

$$
\lambda^{*}<\bar{\lambda}<\lambda
$$

such that $(9)_{\bar{\lambda}}$ possesses a positive solution, $w_{\bar{\lambda}}$. Since $\bar{\lambda}<\lambda$, $w_{\bar{\lambda}}$ is a subsolution of $(9)_{\lambda}$.

On the other hand, a constant $K>0$ large enough satisfying (26) and $K>w_{\bar{\lambda}}$ is a supersolution. Consequently, $(9)_{\lambda}$ possesses a positive solutions, for all $\lambda>\lambda^{*}$.

If $\Omega_{a 0} \neq \emptyset$ and the bifurcation direction at $\lambda_{a 0}$ is subcritical or $\Omega_{a 0}=\emptyset$, we need to show existence of positive solution for $\lambda=\lambda^{*}$. Indeed, in both cases we have

$$
\lambda^{*}<\lambda_{a 0} .
$$

Thus, let $\sigma_{n}$ be a minimizer sequence such that $\sigma_{n} \downarrow \lambda^{*}$ and $w_{n}$ a respective positive solution. Then $w_{n}$ is bounded in $\mathcal{C}(\bar{\Omega})$. Since $\sigma_{1}>\lambda_{1}$ and $\sigma_{n} \leq \sigma_{1}$, Corollary 1 gives

$$
w_{n} \leq \theta_{\sigma_{1}} \quad \forall n \in \mathbf{N},
$$


where $\theta_{\sigma_{1}}$ denote the unique solution of (19) with $\lambda=\sigma_{1}$. Thus, $\left\|w_{n}\right\|_{0} \leq$ $\left\|\theta_{\sigma_{1}}\right\|_{0}$.

In addition, once that $\left(\sigma_{n}, w_{n}\right)$ is a solution of $(9)_{\sigma_{n}}$, we have

$$
\int_{\Omega} \nabla w_{n} \cdot \nabla \phi=\int_{\Omega}\left(\sigma_{n} q\left(x, w_{n}\right)-b q\left(x, w_{n}\right)^{p}\right) \phi \quad \forall \phi \in H_{0}^{1}(\Omega)
$$

Taking $\phi=w_{n}$ as a test function and using (10) we derive that

$$
\begin{aligned}
\left\|w_{n}\right\|_{H_{0}^{1}}^{2} & =\int_{\Omega}\left(\sigma_{n} q\left(x, w_{n}\right)-b q\left(x, w_{n}\right)^{p}\right) w_{n} \\
& \leq \sigma_{1} \int_{\Omega} q\left(x, w_{n}\right) w_{n} \leq \sigma_{1} \int_{\Omega} w_{n}^{2} \leq \sigma_{1}\left\|\theta_{\sigma_{1}}\right\|_{0}^{2}|\Omega| .
\end{aligned}
$$

As a consequence, $w_{n}$ is bounded in $H_{0}^{1}(\Omega)$. Thus, up to a subsequence if necessary,

$$
w_{n} \rightarrow w^{*} \text { in } H_{0}^{1}(\Omega) \quad \text { and } \quad w_{n} \rightarrow w^{*} \text { in } L^{m}(\Omega) \quad m<2^{*} .
$$

Passing to the limit $n \rightarrow \infty$ in (28), it yields

$$
\int_{\Omega} \nabla w^{*} \cdot \nabla \phi=\int_{\Omega}\left(\lambda^{*} q\left(x, w^{*}\right)-b q\left(x, w^{*}\right)^{p}\right) \phi \quad \forall \phi \in H_{0}^{1}(\Omega) .
$$

Hence $w^{*}$ is a weak solution of $(9)_{\lambda^{*}}$ and by the elliptic regularity, we obtain that $w^{*}$ is a classical non-negative solution. We claim that $w^{*} \neq 0$. Indeed, otherwise by elliptic regularity and the Morrey theorem, we have

$$
\left\|w_{n}\right\|_{\mathcal{C}^{1}(\bar{\Omega})} \leq C
$$

for some positive constant $C$. Thus, by the compact embeddeding of $\mathcal{C}^{1}(\bar{\Omega})$ into $\mathcal{C}(\bar{\Omega})$, up to a subsequence if necessary, we deduce that

$$
\left\|w_{n}\right\|_{0} \rightarrow 0
$$

In view of (11), for all $\delta>0$, there exists $n_{\delta} \in \mathbb{N}$ such that

$$
\frac{q\left(x, w_{n}\right)}{w_{n}}-\mathcal{X}_{\Omega_{a 0}}(x) \leq \delta \quad \forall n>n_{\delta}, x \in \Omega .
$$

Consequently,

$$
0=\lambda_{1}\left[-\Delta-\sigma_{n} \frac{q\left(x, w_{n}\right)}{w_{n}}+b \frac{q\left(x, w_{n}\right)^{p}}{w_{n}} ; \Omega\right]>\lambda_{1}\left[-\Delta-\sigma_{n}\left(\delta+\mathcal{X}_{\Omega_{a 0}} ; \Omega\right)\right]
$$

Taking $\delta \rightarrow 0$ imply $n \rightarrow \infty$ and we deduce that

$$
0 \geq \lambda_{1}\left[-\Delta-\lambda^{*} \mathcal{X}_{\Omega_{a 0}} ; \Omega\right]=\mu\left(\lambda^{*}\right) .
$$

By the properties of $\mu$ (see Proposition 1), the above inequality provides us that $\lambda^{*} \geq \lambda_{a 0}$, which is a contradiction with (27). 
To complete the proof, it remains to show that $\lambda_{1}<\lambda^{*} \leq \lambda_{a 0}$. Indeed, If $\Omega_{a 0}=\emptyset$ then $\lambda_{a 0}=\infty$ and $\lambda^{*} \leq \lambda_{a 0}$ is immediate. If $\Omega_{a 0} \neq \emptyset$ then $\lambda_{a 0}$ is a bifurcation point from the trivial solution and, by definition of $\lambda^{*}$, it follows that $\lambda^{*} \leq \lambda_{a 0}$. In order to prove $\lambda_{1}<\lambda$, if $\lambda^{*}<\lambda_{a 0}$, then we have already know, that $(9)_{\lambda}$ possesses a positive solution for $\lambda=\lambda^{*}$ and since $\lambda>\lambda_{1}$ is a necessary condition for the existence, it follows that $\lambda^{*}>\lambda_{1}$. If $\lambda^{*}=\lambda_{a 0}$, since we are considering only the case $a \neq 0$ in $\Omega$, this implies that $\lambda_{1}<\lambda_{a 0}=\lambda^{*}$. Proof of Theorem 3 (a): Recall that, by Corollary 1 , every solution $w>0$ of $(9)_{\lambda}$ satisfies

$$
w \leq\left\|\theta_{\lambda}\right\|_{0}
$$

Thus, let us consider the function

$$
f(x, s):=\lambda q(x, s)-b q(x, s)^{p}+K s .
$$

Since

$$
f_{s}(x, s)=\lambda q_{s}(x, s)-b p q(x, s)^{p-1} q_{s}(x, s)+K \quad \forall s>0,
$$

and $q_{s}(x, s)$ is bounded for $0<s<\left\|\theta_{\lambda}\right\|_{0}$, we can choose $K>0$ large enough such that this function is increasing on $\left[0,\left\|\theta_{\lambda}\right\|_{0}\right]$. Thus, the monotonic interaction

$$
-\Delta w_{n+1}+K w_{n+1}=\lambda q\left(x, w_{n}\right)-b q\left(x, w_{n}\right)^{p}+K w_{n}, \quad w_{0}=\theta_{\lambda}
$$

provides a maximal solution in $\left[0, \theta_{\lambda}\right]$. Once that every positive solution $w>0$ satisfies $w<\theta_{\lambda}$, we get the result.

Now, given $\lambda^{*}(b) \leq \mu<\lambda$, then $W_{\mu}$ is a subsolution of $(9)_{\lambda}$. Since $K>0$ large enough is a super solution of $(9)_{\lambda}$, we derive that $(9)_{\lambda}$ possesses a positive solution $w$ with

$$
W_{\mu}<w \leq K
$$

The strict inequality occurs because $W_{\mu}$ is not a solution of $(9)_{\lambda}$. Once that $W_{\lambda}$ is a maximal solution of $(9)_{\lambda}$, we deduce

$$
W_{\mu}<w \leq W_{\lambda}
$$

This completes the proof.

In order to prove (7), we need the following result

Lemma 2 If $b_{1}<b_{2}$, then $\inf \Lambda_{b_{1}} \leq \inf \Lambda_{b_{2}}$.

Proof Just note that $\Lambda_{b_{2}} \subset \Lambda_{b_{1}}$. Indeed, if $\lambda \in \Lambda_{b_{2}}$, then $w_{\lambda\left(b_{2}\right)}$ is a subsolution of $(9)_{\lambda}$ with $b=b_{1}$. Choosing $K$ large enough satisfying (26) and $K \geq w_{\lambda\left(b_{2}\right)}$, it follows that there exists a positive solution of $(9)_{\lambda}$ with $b=b_{1}$. Moreover,

$$
w_{\lambda\left(b_{2}\right)} \leq w_{\lambda\left(b_{1}\right)} \text {. }
$$


Proof of Theorem 3 (b): Fix $\lambda>\lambda_{1}$, we can choose $\lambda=\lambda_{1}+\varepsilon_{0}$, with $\varepsilon_{0}>0$. Let be $C>0$ a constant, then $\underline{w}=C \varphi_{1}^{m}$ is a subsolution of $(9)_{\lambda}$ if

$$
\begin{aligned}
& C m(1-m)\left|\nabla \varphi_{1}\right|^{2} \frac{\varphi_{1}^{m-2}}{q\left(x, C \varphi_{1}^{m}\right)}+\lambda_{1}\left(m \frac{C \varphi_{1}^{m}}{q\left(x, C \varphi_{1}^{m}\right)}-1\right) \\
& +b q\left(x, C \varphi_{1}^{m}\right)^{p-1} \leq \varepsilon_{0},
\end{aligned}
$$

for all $x \in \Omega$. Let us obtain conditions for that (29) is fulfilled in $\Omega_{\delta}$ as well as in $\Omega \backslash \Omega_{\delta}$, where $\Omega_{\delta}$ is given as in (24).

Firstly, fix $m=m(\lambda)>1$ such that

$$
\lambda_{1}(m-1)<\frac{\epsilon_{0}}{2}
$$

For this $m$, we pick $\delta=\delta(m)$ as in Proposition 4. Observe that $\delta$ does not depend on $C$.

Now, recall that the map $s \mapsto q(x, s) / s$ is increasing and $\lim _{s \rightarrow \infty} q(x, s) / s=1$ (see Lemma 1), therefore

$$
\frac{s}{q(x, s)} \downarrow 1 \text { as } s \rightarrow \infty
$$

Since

$$
\min _{\Omega \backslash \Omega_{\delta}} \varphi_{1}^{m}>0
$$

from (30) and the above limit, we can get $C>0$ large such that

$$
\lambda_{1}\left(m \frac{C \varphi_{1}^{m}}{q\left(x, C \varphi_{1}^{m}\right)}-1\right) \leq \frac{\varepsilon_{0}}{2} \quad \forall x \in \Omega \backslash \Omega_{\delta} .
$$

As a consequence, for $b>0$ satisfying

$$
b q\left(x, C \varphi_{1}^{m}\right)^{p-1} \leq \frac{\varepsilon_{0}}{2} \quad \forall x \in \Omega,
$$

we derive that (29) occurs for all $x \in \Omega \backslash \Omega_{\delta}$.

On the other hand, if $x \in \Omega_{\delta}$ we have

$$
m(1-m)\left|\nabla \varphi_{1}\right|^{2} \varphi_{1}^{m-2}+m \lambda_{1} \varphi_{1}^{m} \leq 0
$$

implying

$$
C m(1-m)\left|\nabla \varphi_{1}\right|^{2} \frac{\varphi_{1}^{m-2}}{q\left(x, C \varphi_{1}^{m}\right)}+m \lambda_{1} \frac{C \varphi_{1}^{m}}{q\left(x, C \varphi_{1}^{m}\right)} \leq 0 .
$$

In view of (31), it follows that (29) also meets in $\Omega_{\delta}$ and therefore $\underline{w}=C \varphi_{1}^{m}$ is a subsolution of $(9)_{\lambda}$. Taking $K$ satisfying (26) and $K \geq C \varphi_{1}^{m}$ it is a supersolution of $(9)_{\lambda}$. Hence,

$$
C \varphi_{1}^{m} \leq w_{[\lambda, b]} \leq K .
$$

As a consequence, given $\varepsilon>0$, there exists $b_{\varepsilon}>0$ such that

$$
\lambda_{1}<\lambda^{*}\left(b_{\varepsilon}\right) \leq \lambda_{1}+\varepsilon
$$

by Proposition 2 , the above inequality is verified for all $0<b \leq b_{\varepsilon}$, showing (7). 
Proposition 5 Let $\left(w_{\lambda^{*}(b)}\right)_{b>0}$ be a family of positive solutions, then

$$
\lim _{b \rightarrow 0}\left\|w_{\lambda^{*}(b)}\right\|_{0}=\infty
$$

Proof Arguing by contradiction, suppose that $\left\|w_{\lambda^{*}(b)}\right\|_{0} \leq M$, for each $b<b_{0}$. Hence

$$
\begin{array}{r}
0=\lambda_{1}\left[-\Delta-\lambda^{*}(b) \frac{q\left(x, w_{\lambda^{*}(b)}\right)}{w_{\lambda^{*}(b)}}+b \frac{q\left(x, w_{\lambda^{*}(b)}\right)^{p}}{w_{\lambda^{*}(b)}} ; \Omega\right] \\
\geq \lambda_{1}\left[-\lambda^{*}(b) \frac{q(x, M)}{M} ; \Omega\right]
\end{array}
$$

Letting to $b \rightarrow 0$, yields

$$
0 \geq \lambda_{1}\left[-\Delta-\lambda_{1} \frac{q(x, M)}{M} ; \Omega\right] .
$$

Since $\Omega_{a 0} \neq \Omega$, then $q(x, M) / M<1$ and it imply

$$
0>\lambda_{1}\left[-\Delta-\lambda_{1} ; \Omega\right]=0
$$

which is a contradiction.

As a consequence of this result, we get

Proof of Theorem 3 (c): By Theorem 3 (a), for all $b>0$ we have

$$
w_{\lambda^{*}(b)} \leq W_{\lambda^{*}(b)} \leq W_{\lambda(b)} .
$$

Thus, by the Proposition 5, we obtain the result.

\section{Multiplicity of positive solutions}

This section is dedicated to obtain a second positive solution of $(9)_{\lambda}$ and for this propose, we use variational methods. The arguments presented here are inspired by [1] and [2].

For each $\lambda>\lambda_{1}$, let $M>0$ be such that $\left\|\theta_{\lambda}\right\|_{0}<M$ where $\theta_{\lambda}$ is stands for the unique solutions of (19), see Proposition 2. Fix $\varepsilon>0$, we define

$$
\bar{q}(x, s)= \begin{cases}q(x, s) & \text { if } s \leq M \\ \phi(x, s) & \text { if } M \leq s \leq M+\varepsilon \\ q(x, M+\varepsilon) & \text { if } M+\varepsilon<s\end{cases}
$$

where $\phi(x, s)$ is a regular function such that the map $s \in(0, \infty) \mapsto \bar{q}(x, s)$ is of class $\mathcal{C}^{1}$. Defining the functional $I_{\lambda}: H_{0}^{1}(\Omega) \rightarrow \mathbb{R}$ given by

$$
I_{\lambda}(w)=\frac{1}{2}\|w\|_{H_{0}^{1}}^{2}-\lambda \int_{\Omega} Q(x, w) d x+b \int_{\Omega} Q_{p}(x, w) d x
$$


where

$$
Q(x, w):=\int_{0}^{w} \bar{q}(x, s) d s \quad \text { and } \quad Q_{p}(x, w):=\int_{0}^{w} \bar{q}(x, s)^{p} d s .
$$

Thus, $I_{\lambda}$ is well-defined and of class $\mathcal{C}^{2}$, for all $\lambda>\lambda_{1}$. Moreover, since every positive solution of $(9)_{\lambda}$ is bounded from above by $M$ (according to Corollary 1 ), then critical points of $I_{\lambda}$ are weak positive solutions of $(9)_{\lambda}$ and by elliptic regularity, are classical solution of $(9)_{\lambda}$

Let us collect some properties of this functional.

Proposition 6 The functional $I_{\lambda}$ is coercive and bounded from below.

Proof For each $w \in H_{0}^{1}(\Omega)$ we have

$$
\begin{aligned}
I_{\lambda}(w) & =\frac{1}{2}\|w\|_{H_{0}^{1}}^{2}-\lambda \int_{\Omega} Q(x, w) d x+b \int_{\Omega} Q_{p}(x, w) d x \\
& =\frac{1}{2}\|w\|_{H_{0}^{1}}^{2}-\int_{\Omega} \int_{0}^{w}\left(\lambda \bar{q}(x, w)-b \bar{q}(x, w)^{p}\right) d s d x
\end{aligned}
$$

since the map

$$
s \mapsto \lambda s-b s^{p}, s \geq 0
$$

is bounded above, we can obtain a constant $C>0$ such that

$$
\lambda \bar{q}(x, s)-b \bar{q}(x, s)^{p} \leq C, \quad s \geq 0 .
$$

In this way, we get

$$
I_{\lambda}(w) \geq \frac{1}{2}\|w\|_{H_{0}^{1}}^{2}-C \int_{\Omega} w d x \geq \frac{1}{2}\|w\|_{H_{0}^{1}}^{2}-C|w|_{1} .
$$

By the continuous embedding $H_{0}^{1}(\Omega) \hookrightarrow L^{1}(\Omega)$ it follows

$$
I_{\lambda}(w) \geq \frac{1}{2}\|w\|_{H_{0}^{1}}^{2}-C_{1}\|w\|_{H_{0}^{1}} .
$$

Showing that $I_{\lambda}$ is coercive and bounded below.

Proposition 7 If $w_{n}$ is a sequence in $H_{0}^{1}(\Omega)$ with $I_{\lambda}\left(w_{n}\right)$ bounded, then, up a subsequence if necessary,

$$
w_{n} \rightarrow w \text { in } H_{0}^{1}(\Omega)
$$

and

$$
I_{\lambda}(w) \leq \liminf _{n \rightarrow \infty} I_{\lambda}\left(w_{n}\right) .
$$

In particular, $I_{\lambda}$ attains its infimum on $H_{0}^{1}(\Omega)$.

coercive 
Proof Thanks to the coercivity of $I_{\lambda}$, the sequence $w_{n}$ is bounded in $H_{0}^{1}(\Omega)$.

Thus, up to a subsequence if necessary,

$$
w_{n} \rightarrow w \text { in } H_{0}^{1}(\Omega)
$$

and

$$
w_{n} \rightarrow w \text { in } L^{s}(\Omega), s \in\left[1,2^{*}\right)
$$

Consequently,

$$
\begin{aligned}
I_{\lambda}(w)-I_{\lambda}\left(w_{n}\right)= & \frac{1}{2}\left(\|w\|_{H_{0}^{1}}^{2}-\left\|w_{n}\right\|_{H_{0}^{1}}^{2}\right)+ \\
& \int_{\Omega}^{2}\left[\left(\lambda Q\left(x, w_{n}\right)-b Q_{p}\left(x, w_{n}\right)\right)-\left(\lambda Q(x, w)-b Q_{p}(x, w)\right)\right] d x .
\end{aligned}
$$

Writing $F(x, s)=\lambda Q(x, s)-b Q_{p}(x, s), s \geq 0$, we have

$$
I_{\lambda}(w)-I_{\lambda}\left(w_{n}\right)=\frac{1}{2}\left(\|w\|_{H_{0}^{1}}^{2}-\left\|w_{n}\right\|_{H_{0}^{1}}^{2}\right)+\int_{\Omega}\left[F\left(x, w_{n}\right)-F(x, w)\right] d x .
$$

By the properties of $\bar{q}$,

$$
F_{s}(x, s)=\lambda \bar{q}(x, s)-b \bar{q}(x, s)^{p}
$$

is bounded in $\Omega \times[0, \infty)$. Thus, (34) implies

$$
\begin{aligned}
& I_{\lambda}(w)-I_{\lambda}\left(w_{n}\right)=\frac{1}{2}\left(\|w\|_{H_{0}^{1}}^{2}-\left\|w_{n}\right\|_{H_{0}^{1}}^{2}\right)+ \\
& \int_{\Omega}\left[\int_{0}^{1}\left(\lambda \bar{q}\left(x, t w_{n}+(1-t) w\right)-b \bar{q}\left(x, t w_{n}+(1-t) w\right)^{p} d t\left(w_{n}-w\right)\right] d x\right. \\
& \leq \frac{1}{2}\left(\|w\|_{H_{0}^{1}}^{2}-\left\|w_{n}\right\|_{H_{0}^{1}}^{2}\right)+C \int_{\Omega}\left|w_{n}-w\right| d x
\end{aligned}
$$

Since $w_{n} \rightarrow w$ in $L^{1}(\Omega)$ and $w_{n} \rightarrow w$ in $H_{0}^{1}(\Omega)$, it follows

$$
I_{\lambda}(w)-\liminf _{n \rightarrow \infty} I_{\lambda}\left(w_{n}\right) \leq 0
$$

Finally, since $I_{\lambda}$ is coercive and bounded below (Proposition 6), we obtain $I_{\lambda}$ attains its infimum on $H_{0}^{1}(\Omega)$.

In order to apply Theorem II.11.8 of [12], let us prove that $I_{\lambda}$ has two solutions that are local minimum of $I_{\lambda}$ in $H_{0}^{1}(\Omega)$.

Proposition 8 For all $\lambda>\lambda^{*},(9)_{\lambda}$ possesses a solution $w$ that is a local minimum for $I_{\lambda}$ in $H_{0}^{1}(\Omega)$.

Proof By Theorem 3 (a), the maximal solution of $(9)_{\lambda^{*}}, W_{\lambda^{*}}$, is a strict subsolution of $(9)_{\lambda}$ for all $\lambda>\lambda^{*}$. Thus, we obtain a solution $v_{\lambda}$ for $(9)_{\lambda}$ via minimization

$$
I_{\lambda}\left(v_{\lambda}\right)=\inf \left\{I_{\lambda}(w) ; w \in H_{0}^{1}(\Omega), w(x) \geq W_{\lambda^{*}}\right\} .
$$


Hence, $v_{\lambda}$ exists thanks to Propositions 6 and 7 and it defines a solution to $(9)_{\lambda}$.

To verify that it is a minimizer of $I_{\lambda}$ in $H_{0}^{1}(\Omega)$, by [4] it suffices to show that is a local minimizer in the $\mathcal{C}^{1}$ topology.

Taking $K>0$ sufficiently large such that $s \mapsto \lambda \bar{q}(x, s)-b \bar{q}(x, s)^{p}+K s$ be increasing in $\left[0, \max _{\bar{\Omega}} v_{\lambda}\right]$ and since $v_{\lambda}>W_{\lambda^{*}}$, we derive that

$$
\begin{aligned}
-\Delta\left(v_{\lambda}-W_{\lambda^{*}}\right)+K\left(v_{\lambda}-W_{\lambda^{*}}\right) & =\left(\lambda \bar{q}\left(x, v_{\lambda}\right)-b \bar{q}\left(x, v_{\lambda}\right)^{p}+K v_{\lambda}\right) \\
& -\left(\lambda^{*} \bar{q}\left(x, W_{\lambda^{*}}\right)-b \bar{q}\left(x, W_{\lambda^{*}}\right)^{p}+K W_{\lambda^{*}}\right)>0 .
\end{aligned}
$$

By the Strong Maximum Principle, it follows that $v_{\lambda}-W_{\lambda^{*}}$ lies in the interior of the positive cone of $\mathcal{C}_{0}^{1}(\bar{\Omega})$. Hence, there exists $\varepsilon>0$ such that

$$
B_{\varepsilon}\left(v_{\lambda}\right) \subset\left\{u \in \mathcal{C}_{0}^{1}(\bar{\Omega}) ; u \geq W_{\lambda^{*}}\right\},
$$

where $B_{\varepsilon}\left(v_{\lambda}\right)$ denote the open ball of radius $\varepsilon$ and center $v_{\lambda}$ in $\mathcal{C}^{1}$ topology.

Since $I_{\lambda}\left(v_{\lambda}\right)$ is the minimizer in $\left\{u \in H_{0}^{1}(\Omega) ; u \geq W_{\lambda^{*}}\right\}$, then it is also a local minimizer in $\mathcal{C}_{0}^{1}(\Omega)$.

The next result gives us a second local minimum of $I_{\lambda}$ in $H_{0}^{1}(\Omega)$.

Proposition 9 If $\lambda<\lambda_{a 0}$, then the trivial solution $w \equiv 0$ is a local minimum of $I_{\lambda}$ on $H_{0}^{1}(\Omega)$ and is an isolated solution of $(9)_{\lambda}$.

Proof We will consider two cases:

Case $\Omega_{a 0} \neq \emptyset$

$\overline{\text { Fix } \varepsilon=\varepsilon(\lambda)}>0$ sufficiently small such that

$$
1-\varepsilon \frac{\lambda}{\lambda_{1}}-\frac{\lambda}{\lambda_{a 0}}>0 .
$$

Then, thanks to the properties of $\bar{q}$, we can get $C>0$ and $1<r<2^{*}$ such that

$$
\bar{q}(x, s) \leq q(x, s) \leq\left(\varepsilon+\mathcal{X}_{\Omega_{a 0}}(x)\right) s+C s^{r} \quad \forall(x, s) \in \Omega \times[0, \infty) .
$$

Consequently,

$$
\begin{aligned}
I_{\lambda}(w) & \geq \frac{1}{2}\|w\|_{H_{0}^{1}}^{2}-\frac{\lambda}{2} \int_{\Omega}\left(\varepsilon+\mathcal{X}_{\Omega_{a 0}}(x)\right) w^{2}-\frac{C}{r+1} \int_{\Omega} w^{r+1} \\
& \geq \frac{1}{2}\left(1-\varepsilon \frac{\lambda}{\lambda_{1}}-\frac{\lambda}{\lambda_{a 0}}\right)\|w\|_{H_{0}^{1}}^{2}-\frac{C}{\lambda_{1}(r+1)}\|w\|_{H_{0}^{1}}^{r+1} .
\end{aligned}
$$

Therefore, there exists $\delta>0$ small such that

$$
I_{\lambda}(w) \geq 0 \quad \forall w \in H_{0}^{1}(\Omega),\|w\|_{H_{0}^{1}} \leq \delta,
$$

showing that $w \equiv 0$ is a local minimum of $I_{\lambda}$ in $H_{0}^{1}(\Omega)$.

To prove that 0 is isolated solution of (9) we argue by contradiction. Otherwise, there would be a sequence of positive solution $w_{n}$ such that $\left\|w_{n}\right\|_{H_{0}^{1}} \rightarrow 0$. 
Therefore, we also have $\left\|w_{n}\right\|_{0} \rightarrow 0$. By (11), for all $\delta>0$, exists $n_{\delta} \in \mathbb{N}$ such that

$$
\frac{q\left(x, w_{n}\right)}{w_{n}}-\mathcal{X}_{\Omega_{a 0}} \leq \delta \quad \forall n>n_{\delta}, x \in \Omega .
$$

Consequently,

$$
0=\lambda_{1}\left[-\Delta-\lambda \frac{q\left(x, w_{n}\right)}{w_{n}}+b \frac{q\left(x, w_{n}\right)^{p}}{w_{n}} ; \Omega\right]>\lambda_{1}\left[-\Delta-\lambda\left(\delta+\mathcal{X}_{\Omega_{a 0}}\right) ; \Omega\right]
$$

Taking $\delta \rightarrow 0$ we deduce that

$$
0 \geq \lambda_{1}\left[-\Delta-\lambda \mathcal{X}_{\Omega_{a 0}} ; \Omega\right]=\mu(\lambda)
$$

By the properties of $\mu$ (see Proposition 1), the above inequality provides us $\lambda \geq \lambda_{a 0}$, which is a contradiction.

Case $\Omega_{a 0}=\emptyset$

Similarly, using $q(x, s) \leq s$, we have

$$
\begin{aligned}
I_{\lambda}(w) & \geq \frac{1}{2}\|w\|_{H_{0}^{1}}^{2}-\frac{\lambda}{2} \int_{\Omega} w^{2} \\
& \geq \frac{1}{2}\left(1-\frac{\lambda}{\lambda_{1}}\right)\|w\|_{H_{0}^{1}}^{2} .
\end{aligned}
$$

implying that 0 is a local minimum of $I_{\lambda}$ in $H_{0}^{1}(\Omega)$. Moreover, observing that $\mathcal{X}_{\Omega_{a 0}} \equiv 0$, the same arguments of previous case can be applied to conclude that 0 is an isolated solution of (9).

Recall that, according to Definition II.12.2 in [12], for a convex and closed set $M \subset H_{0}^{1}(\Omega)$, a function $w \in H_{0}^{1}(\Omega)$ is a critical point of $I_{\lambda}$ on $M$ if

$$
g(w)=\sup \left\{I_{\lambda}^{\prime}(w)(w-v) ; v \in M,\|v-w\|_{H_{0}^{1}} \leq 1\right\}=0 .
$$

Taking

$$
\mathcal{M}=\left\{w \in H_{0}^{1}(\Omega) ; 0 \leq w(x) \leq v_{\lambda}(x)\right\} .
$$

Since $w \equiv 0$ and $v_{\lambda}$ are solutions of (9), then a critical point of $I_{\lambda}$ in $\mathcal{M}$ is also a critical of $I_{\lambda}$ in $H_{0}^{1}(\Omega)$. Let us show a Palais-Smale condition for the functional $I$ in $\mathcal{M}$.

Proposition 10 If $w_{n}$ is a sequence in $\mathcal{M}$ such that

$$
I_{\lambda}\left(w_{n}\right) \rightarrow c \quad \text { and } \quad g\left(w_{n}\right) \rightarrow 0
$$

then $w_{n}$ possesses a strongly convergent subsequence in $H_{0}^{1}(\Omega)$. 
Proof

$$
w_{n} \rightarrow w \text { in } H_{0}^{1}(\Omega) \quad \text { and } \quad w_{n}(x) \rightarrow w(x) \text { a.e. in } \Omega \text {. }
$$

Once that $0 \leq w_{n} \leq v_{\lambda}$, we obtain $0 \leq w \leq v_{\lambda}$ and from Lebesgue's Dominated Convergence Theorem we get

$$
\int_{\Omega}\left(\lambda \bar{q}\left(x, w_{n}\right)-b \bar{q}\left(x, w_{n}\right)^{p}\right)\left(w_{n}-w\right) d x \rightarrow 0
$$

Therefore,

$$
\begin{aligned}
g\left(w_{n}\right)\left\|w_{n}-w\right\|_{H_{0}^{1}} & \geq I_{\lambda}^{\prime}\left(w_{n}\right)\left(w_{n}-w\right) \\
& =\int_{\Omega} \nabla w_{n} \nabla\left(w_{n}-w\right)+o(1) \\
& =\int_{\Omega}\left|\nabla\left(w_{n}-w\right)\right|^{2}+o(1) .
\end{aligned}
$$

Thus,

$$
g\left(w_{n}\right) \geq\left\|w_{n}-w\right\|_{H_{0}^{1}}+o(1)
$$

Passing to the limit $n \rightarrow \infty$ we deduce that $w_{n} \rightarrow w$ in $H_{0}^{1}(\Omega)$.

Finally, we are able to give the

Proof of Theorem 2 (c): Consider again the set

$$
\mathcal{M}=\left\{w \in H_{0}^{1}(\Omega) ; 0 \leq w(x) \leq v_{\lambda}(x)\right\}
$$

where $v_{\lambda}$ is a solution that is a local minimum of $I_{\lambda}$ on $\mathcal{M}$ (according Proposition 8). Once that $I_{\lambda}$ satisfies the Palais-Smale condition in $\mathcal{M}$ (Proposition 10), we can apply the Theorem II.11.8 of [12] and deduce the following dichotomy: either

1. $I_{\lambda}$ has a critical point $w_{\lambda}$ in $\mathcal{M}$ which is not a local minimum; or

2. $I_{\lambda}\left(v_{\lambda}\right)=I_{\lambda}(0)$ and $v_{\lambda}$ and 0 may be connected in any neighborhood of the set of local minimal of $I_{\lambda}$ relative to $\mathcal{M}$, each of which satisfying $I_{\lambda}(w)=0$

But, by Proposition 9,0 is an isolated among the solution of $(9)_{\lambda}$, for all $\lambda \in\left(\lambda_{1}, \lambda_{a 0}\right)$. This excludes the possibility of the paragraph 2 . occurs.

Acknowledgements WC is Bolsista da CAPES Proc. no BEX 6377/15-7. CMR and AS have been partially supported for the project MTM2015-69875-P (MINECO/FEDER, UE) and AS by the project CNPQ-Proc. 400426/2013-7 . 


\section{References}

1. Alama, S. and Tarantello, G., Elliptic problems with nonlinearities indefinite in sign, J. Funct. Anal., 141, 159-215 (1996).

2. Ambrosetti, A. and Brezis, H. and Cerami, G., Combined effects of concave and convex nonlinearities in some elliptic problems, J. Funct. Anal., 122, 519-543 (1994).

3. Arcoya, D. and Carmona, J. and Pellacci, B., Bifurcation for some quasilinear operators Proc. Roy. Soc. Edinburgh Sect. A, 131, 733-765 (2001).

4. Brezis, H. and Nirenberg, L., $H^{1}$ versus $C^{1}$ local minimizers, C. R. Acad. Sci. Paris Sér. I Math., 317, 465-472 (1993).

5. Brezis, H. and Oswald, L., Remarks on sublinear elliptic equations, Nonlinear Anal., 10, 55-64 (1986).

6. Cantrell, R. S. and Cosner, C., Diffusive logistic equations with indefinite weights: population models in disrupted environments, Proc. of the Royal Soc. of Edinburgh, 112 A, 293-318 (1989).

7. de Figueiredo, D. G., Positive solutions of semilinear elliptic problems, Lecture Notes in Math., 957, 34-87 (1982).

8. Delgado, M. and López-Gómez, J. and Suárez, A., Combining linear and nonlinear diffusion, Adv. Nonlinear Stud., 4, 273-287 (2004)

9. Hess, P. and Kato, T., On some linear and nonlinear eigenvalue problems with an indefinite weight function, Comm. Partial Differential Equations, 5, 999-1030 (1980).

10. López-Gómez, J., The maximum principle and the existence of principal eigenvalues for some linear weighted boundary value problems, J. Differential Equations, 127, 263-294 (1996)

11. Ouyang, T., On the positive solutions of semilinear equations $\Delta u+\lambda u-h u^{p}=0$ on the compact manifolds, Trans. Amer. Math. Soc., 331, 503-527 (1992).

12. Struwe M., Variational Method, Springer, (1990). 